\title{
Generating Seizure-Inducing Sequences with Interactive Visualizations
}

\author{
Laura South * Michelle A. Borkin ${ }^{\dagger}$
}

Northeastern University

\begin{abstract}
Interactive visualizations are often built to draw the eye towards pertinent information with attention-grabbing pops of color and patterns. These techniques, though helpful in engaging the average user and nudging them towards important information, can be harmful to users with photosensitive epilepsy, who may experience seizures when exposed to content with flashes, transitions to and from saturated red, or repeated patterns. In this paper, we explore three case studies of interactive visualizations created without malicious intent yet capable of producing seizure-inducing sequences through interaction alone. Based on these case studies as well as relevant related literature, we contribute a set of simple recommendations to help visualization designers and developers avoid accidentally creating interactive visualizations with the potential to cause seizures.
\end{abstract}

\section{INTRODUCTION AND RELATED WORK}

Photosensitive epilepsy is a condition characterized by seizures triggered by specific sequences of flashing lights or repeated patterns. Of the 65 million individuals with epilepsy worldwide, approximately $14 \%$ experience seizures related to photosensitive risk factors [3]. Over the past fifteen years, a troubling trend has emerged involving malicious attacks targeting individuals with photosensitive epilepsy. In these malicious attacks, an anonymous user sends a flashing or strobing sequence to a photosensitive target with the intent to cause a seizure or similar detrimental effect. Targets have ranged from a journalist discussing controversial topics [5] to support networks for people with epilepsy $[6,7,9]$.

Most reported incidents have taken place on social media platforms or support forums, where attackers can easily send messages containing links to animated images or videos, but these remote attacks can occur in any online environment, including interactive visualizations. The visualization community has discussed accessibility in terms of color-blindness [1] and other visual impairments [4], but less attention has been paid to neurological impairments such as photosensitive epilepsy. Conti, Ahmahad \& Stasko found in 2005 that network visualizations could create seizure-inducing flashes when visualizing artificial datasets generated with the intent to create strobe effects, demonstrating for the first time the potential connection between seizure-inducing content and data visualization [2]. Data visualizations today are more interactive, more collaborative, and appear on a wider range of devices than in 2005. When interactive visualizations are collaborative or appear in public, users sacrifice a degree of control over the system. In such cases, an attacker would no longer need to generate and inject artificial data to produce a seizure-inducing sequence; they would simply need to find a way to interact with the visualization to produce a photosensitive hazard.

There are three primary risk factors present in most seizureinducing sequences: flashes, transitions to and from saturated red, or repeated patterns [3]. All three photosensitive risk factors could reasonably appear in data visualizations. For example, transitions

\footnotetext{
*e-mail: south.1@northeastern.edu
}

$\dagger$ e-mail: m.borkin@northeastern.edu in interactive graphs triggered in rapid succession can form a dangerous flicker effect if there is a significant change in luminance between states. Similarly, the bright red that is often used to produce a "pop-out effect" in visualizations can be dangerous if it covers a large area of the visualization and is attached to an interactive transition. Parallel edges in node-link diagrams can create harmful repeated patterns of light-dark stripes, particularly if the edges are dynamic and can be moved through interaction.

In this work, we explore three case studies of publicly-available interactive visualizations. We tested a screen-recording of interactions with each visualization using the Photosensitive Epilepsy Analysis Tool (PEAT) ${ }^{1}$, an established system for measuring photosensitive risk in videos (screen recordings and PEAT reports are included in Supplementary Material). Although these visualizations were not created with malicious intent, each one has the potential to produce seizure-inducing sequences through interaction alone. This work serves as proof of concept for the very real possibility of malicious attacks against people with photosensitive epilepsy orchestrated using interactive visualizations. In the same way that the visualization community is cognizant of color blindness when picking color maps, we need to be aware of neurological conditions like photosensitive epilepsy when designing interactive and animated visualizations. Although significant future work is necessary for complete protection against this threat, we contribute guidelines to help developers and designers avoid accidentally creating a visualization with the potential to cause seizures.

\section{Case studies}

\subsection{Flashes}

Our first case study is a parallel sets diagram visualizing the demographics and outcomes of passengers and crewmembers aboard the Titanic (Figure 1). An overview is presented first and details are shown when the user hovers over an element. When the details are shown a large area of the visualization sharply brightens to provide visual feedback to the user about their selection. This sharp brightness difference can easily be used to create a flicker or strobe effect by quickly moving the mouse among the items. When analyzing a screen recording of interactions with the visualization with PEAT, we found that the luminance flashes from moving between elements exceeds safety thresholds and creates a photosensitive hazard.
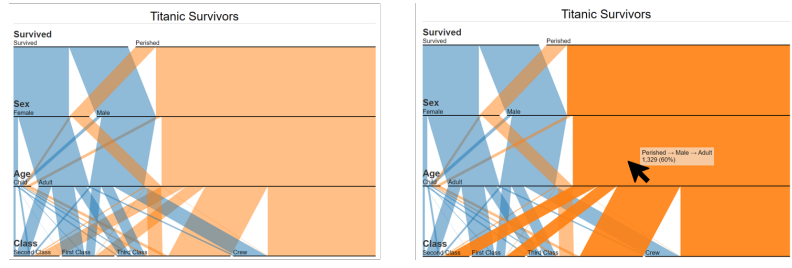

Figure 1: A sharp change in luminance occurs on hover in this visualization of Titanic passengers and crewmembers, creating a flicker effect when the users moves the mouse around quickly. Source: https://www. jasondavies.com/parallel-sets/ 


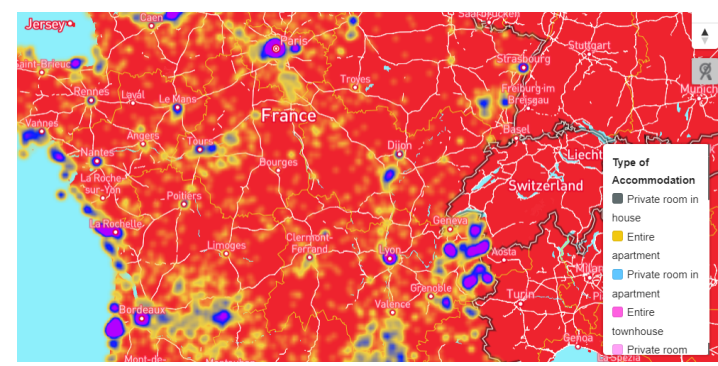

Figure 2: Large areas of this interactive map of Airbnb rentals in Europe are colored with a highly saturated red, producing a dangerous red flicker effect when the user rapidly pans and zooms. Source: https://roqueleal.me/airbnbfr.html

\subsection{Saturated red transitions}

An interactive map of AirBnb rentals in Europe (Figure 2) serves as a good example for harmful transitions to and from saturated red in data visualizations. Land areas on the map are colored according to the most common types of accommodation available. Areas of low population density with little data available are colored red by default. Because large swaths of rural land separate the cities, the map is mostly red. A dangerous red flicker effect can be produced by rapidly panning the map from blue ocean to red land. Analysis with PEAT confirms that the red transitions caused by panning and zooming the map exceed safety thresholds and represent a photosensitive hazard.

\subsection{Repeated patterns}

The Evolution of the American Census is a visual history created by The Pudding, an online data journalism publication. In the visualization (Figure 3), a node-link diagram is used to show how questions have evolved over decades, with similar questions linked by a vertical edge as the user scrolls from 1790 to 2020 . As more questions are introduced to the census in the late 20th century, the node-link diagram begins to resemble a striped pattern. At the visualization's most complex point, the edges form 52 clearly discernible stripes occupying the majority of the screen area. Unfortunately PEAT does not test for repeated patterns, so the photosensitive risk report for this case study does not identify any dangerous content. However, Wilkins et al. defined a dangerous repeated pattern to be one with "clearly discernible stripes where there are more than five light-dark pairs of stripes in any orientation", occupying at least $25 \%$ of the total screen area [8]. Because this visualization incorporates 52 stripes occupying more than $25 \%$ of the screen area, it falls within Wilkins et al.'s definition of a sequence with dangerous repeated patterns.

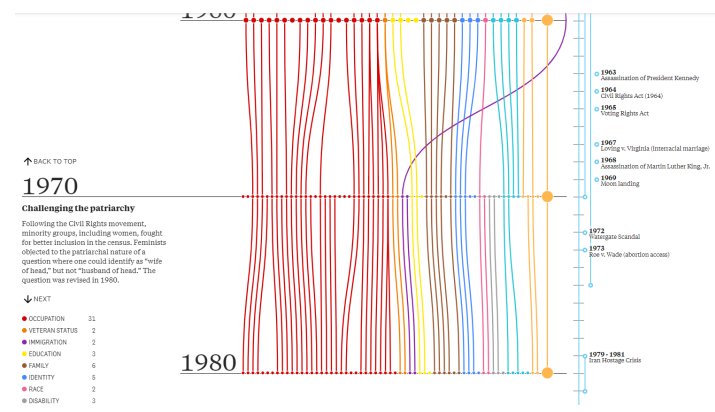

Figure 3: A node-link diagram with 52 edges forms a series of repeated light-dark stripe pairs with the potential to cause seizures when viewed by a photosensitive user. Source: https://pudding.cool/ 2020/03/census-history/

\section{Discussion}

Further work is needed to understand and protect against seizureinducing sequences within interactive visualizations. However, based on our case studies we can recommend simple steps that visualization designers and developers can take to minimize the risk of accidentally creating a photosensitive hazard.

Test your work: Create a screen recording of standard and unusual interactions with your system and test for photosensitive risk factors using a system like PEAT. This is particularly important if the visualization system will be used collaboratively or publicly.

Carefully consider bright colors: Use saturated red and bright colors sparingly on elements that are tied to user interaction. Flashes and red transitions are generally safe if they occupy less than $25 \%$ of the screen area [3].

Give warnings: Insert a warning about potential photosensitive hazards before users begin using the visualization system. Be specific about which risk factors (flashes, red transitions, or patterns) users could be exposed to.

\section{ConCLUSION}

In this paper, we have demonstrated that seemingly innocuous visualizations can be used to produce seizure-inducing sequences through interaction alone. Malicious attacks targeting people with photosensitive epilepsy have taken place on social media with increasing regularity over the past fifteen years, and the visualization community must be aware of the potential for similar attacks using our own tools. Significant work remains to be done in finding ways to detect and mitigate seizure-inducing content in interactive visualizations, but designers and developers can take steps to minimize photosensitive risk in their creations. By making interactive visualizations safer for those with photosensitive epilepsy, we take a step towards the broader goal of creating visualizations that are useful and accessible for all.

\section{ACKNOWLEDGMENTS}

This material is supported by the National Science Foundation Graduate Research Fellowship Program under Grant No. DGE1451070

\section{REFERENCES}

[1] J. Choi, S. Jung, D. G. Park, J. Choo, and N. Elmqvist. Visualizing for the non-visual: Enabling the visually impaired to use visualization. In Computer Graphics Forum, vol. 38, pp. 249-260. Wiley Online Library, 2019.

[2] G. Conti, M. Ahamad, and J. Stasko. Attacking information visualization system usability overloading and deceiving the human. In Proceedings of the 2005 symposium on Usable privacy and security, pp. 89-100, 2005.

[3] R. S. Fisher, G. Harding, G. Erba, G. L. Barkley, and A. Wilkins. Photicand pattern-induced seizures: a review for the epilepsy foundation of america working group. Epilepsia, 46(9):1426-1441, 2005.

[4] D. R. Flatla and C. Gutwin. Individual models of color differentiation to improve interpretability of information visualization. In Proceedings of the SIGCHI Conference on Human Factors in Computing Systems, pp. 2563-2572, 2010

[5] C. Kang. A tweet to Kurt Eichenwald, a strobe, and a seizure. now, an arrest. The New York Times, 2017.

[6] K. Poulsen. Hackers assault epilepsy patients via computer. Wired, Mar. 2008.

[7] N. Swanborough. Epilepsy Society sees worst ever bullying attack on Twitter. Epilepsy Society, May 2020.

[8] A. Wilkins, J. Emmett, and G. Harding. Characterizing the patterned images that precipitate seizures and optimizing guidelines to prevent them. Epilepsia, 46(8):1212-1218, 2005.

[9] E. Wolfe and S. Ahmed. A Twitter cyberattack on the epilepsy foundation posted strobing images that could trigger seizures. CNN Business, Dec. 2019. 